\section{How has the COVID-19 pandemic affect the rejection rates and repeat reasoning of digital radiography?}

Duaa F. Jastaniyyah, MD, Abdulkarim A. Jawhari, MD, Abdullah T. Mugharbel, MD, Mawya A. Khafaji,PhD, MSs, Sarah H. Albahiti, PhD.

\section{ABSTRACT}

Objectives: To compare the digital radiology rejection rates and reasoning pre-COVID-19 (PC) and during COVID-19 (DC) from September 2019 to August 2020.

Methods: This record-base retrospective study where data were extracted from the radiography equipment (Carestream Health, Rochester, New York, USA) at the Department of Radiology, King Abdulaziz University Hospital, Jeddah, Saudi Arabia over 12 months. The data were equally divided into PC and DC.

Results: The DC rejection rate decreased from PC by $2.6 \%$ with a significant $p=0.00001$. During COVID-19 there were 15,376 images of different body parts, versus 23,861 images during PC. Position errors were the main reasons for rejection for both PC (39.8\%) and DC (42.7\%), followed by technique errors (PC: 19.2\%) (DC: 17.2\%).

Conclusion: Different sectors in hospitals were affected by COVID-19, including diagnostic radiology, in the positive direction. Thus, the DC rejection rate was markedly reduced compared with PC. This may be due to radiology technicians becoming more cautious in dealing with patients.

Keywords: reject rate, digital radiography, COVID-19

Saudi Med J 2021; Vol. 42 (9): 1041-1044 doi: 10.15537/smj.2021.42.9.20210254

T $\mathrm{n}$ diagnostic radiology, imaging systems vary and 1 change according to clinical needs, acquisition time, and location. Moreover, the most well-known technological solutions are analog systems, computed radiography (CR), direct radiology (DR) and indirect radiology. Direct radiology systems are commonly used today, replacing most CR systems owing to their immediate availability and increased workflow efficiency. ${ }^{1}$ However, it is fairly uncommon to have pooror suboptimal-quality radiographs, which necessitates repeating the films and thereby increasing the time spent by patients and their exposure to radiation. ${ }^{2}$

In the same way, ionizing radiation can have undesirable effects. These include an increased risk of cancer by damaging molecular bonding that could lead in correct repair, and pregnancy abnormalities, including congenital abnormalities and spontaneous abortions. Therefore, it is mandatory that any $\mathrm{x}$-ray investigation should be justified and optimized. ${ }^{3,4}$ Some of the available quality indicators that can assess the optimization of imaging practice are reject analysis, evaluating protocols, and the use of dose-monitoring tools. ${ }^{5}$ Rejection rate analysis is an important tool that can help ensure a safe radiation culture by making the patient's exposure as low as reasonably achievable. In addition, rejected images lower the adequacy of the department and patient gratification, which, as a result, will raise departmental charges. ${ }^{6}$ World Health Organization recommends a rejection rate of $5 \%$; meanwhile, the Committee on Quality Assurance of the Conference of Radiographic Control Program Directorate $^{7}$ suggests a rejection rate of $10 \%$. Several studies reported a rejection rate ranging from $2.1 \%$ to $33 \%$, and the reasons for these differences include positioning errors, motion errors, or miscellaneous errors. $^{2}$

Coronavirus disease-2019 was first reported in December 2019 in Wuhan, China ${ }^{8}$ and was declared an ongoing pandemic by the WHO in January 2020. It continues to create an impact on an individual, national, and global scale in terms of the economy, increased mortality rate, and socialization due to forced lockdowns to contain the outbreak. ${ }^{9}$ Moreover, healthcare facilities and healthcare workers have been among the most impacted. New guidelines have been published to minimize the spread and ensure safe interactions with COVID-19 and non-COVID-19 patients to minimize contact. ${ }^{10,11}$

This had an impact on all departments, including diagnostic imaging. The effect of pandemics on rejection rates has not been studied previously. Therefore, we aimed to compare the DR rejection rates pre- (PC) and during COVID-19 (DC).

Disclosure. Authors have no conflict of interests, and the work was not supported or funded by any drug company. 
Methods. This record-base retrospective study that was carried out at the Department of Radiology, King Abdulaziz University Hospital, Jeddah, Saudi Arabia, a tertiary center, over a period of 12 months. The period from September 2019 to February 2020 was labelled PC, while March to August 2020 represented the period DC. The categorization was based on the first case reported in Saudi Arabia on March 1, 2020.

Related articles were searched using PubMed engine using keywords including digital radiography, reject rates and COVID-19. The data were extracted from the 6 DR machines (DRX-Evolution; Carestream Health, Rochester, NY, USA) that were included in hospital records. All the machines were in the radiology department, except for one which was in the emergency department. Patients of all ages and of both genders who underwent DR examination during the study period were included.

Statistical analysis. Data entry was performed using Microsoft Excel 2016 (Microsoft Corporation, NM, USA). Statistical Package for Social Sciences version 21 (IBM Corporation, NY, USA) was used in description of the number of images, body part, reason for rejection, and average rejection rate. The comparison between PC and DC rejects rate was carried out using Chi-square test or fisher test for expected count 5. A p-value of $<0.05$ was considered significant. Although all images were included, only body parts that had higher reject rate (PC) were presented in Figure 1. Counting and averaging calculations were performed using the same software.

Rejected images are directly registered within the system, and the technicians chose a reason for rejection before deleting or repeating images. The available
Table 1 - Reasons for rejection of images in direct digital radiography according to period.

\begin{tabular}{lccc}
\hline $\begin{array}{l}\text { Reasons for } \\
\text { rejection }\end{array}$ & Pre-COVID-19 & $\begin{array}{c}\text { During } \\
\text { COVID-19 }\end{array}$ & $P$-value \\
\hline Positioning error & $1338(39.8)$ & $752(42.7)$ & $0.047^{*}$ \\
Technique & $646(19.2)$ & $303(17.2)$ & 0.076 \\
Artifact & $449(13.4)$ & $262(14.9)$ & 0.137 \\
Motion & $376(11.2)$ & $210(11.9)$ & 0.435 \\
Duplicate & $373(11.1)$ & $98(5.6)$ & $0.00001^{*}$ \\
Clipped anatomy & $99(2.9)$ & $26(1.5)$ & $0.001^{*}$ \\
Test/service/blank & $80(2.4)$ & $111(6.3)$ & $0.00001^{*}$ \\
Total & $3361(100)$ & $1762(100)$ & $0.00001^{*}$ \\
\hline \multicolumn{4}{c}{ Values are presented as numbers and percentages (\%). *Significant } \\
\multicolumn{4}{c}{$p$-value using Chi-square test. } \\
\hline \multicolumn{4}{c}{}
\end{tabular}

rejection reasons are predefined in the system as: artifacts, clipped anatomy, duplicates, motion, positioning error, technique, and test/service/blank.

The Institutional Review Board of the Unit of Biomedical Ethics, King Abdulaziz University granted approval to this study (Reference No 41-21).

Results. This study aimed to determine the rejection rate and reasons behind it for DR PC and DC pandemic. Pre-COVID-19 period included 23,861 scans were conducted for both adults and pediatrics, with a rejection rate of $14.1 \%$ for all body parts. On the other hand, only 15,376 radiographs were performed in DC with a rejection rate of $11.5 \%$. The DC reject rate was significantly low compared to $\mathrm{PC}$ period with a $p=0.00001$ and mean difference $=2.6 \%$.

Table 1 present the main reasons for repeats, which are found to be related to radiographers: such as positioning errors and techniques in both $\mathrm{PC}$ and $\mathrm{DC}$

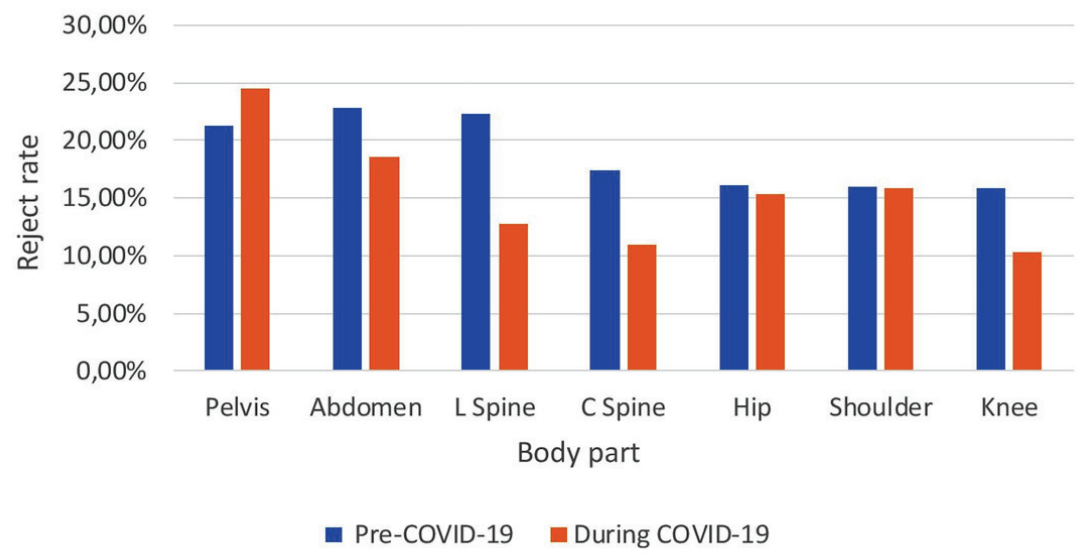

Figure 1 - Rejection rate according to body parts. L spine: lumbar spine, C spine: cervical spine 
Table 2 - Repeat reasons for various body parts pre-COVID-19 and during COVID-19 periods.

\begin{tabular}{|c|c|c|}
\hline Body parts & Pre COVID-19 & During COVID-19 \\
\hline \multicolumn{3}{|l|}{ Abdomen } \\
\hline Artifact & $59(11.3)$ & $44(11.5)$ \\
\hline Clipped anatomy & $20(3.8)$ & $3(0.8)$ \\
\hline Duplicate & $36(6.9)$ & $33(8.6)$ \\
\hline Motion & $29(5.6)$ & $24(6.3)$ \\
\hline Positioning error & $302(58.0)$ & $194(50.9)$ \\
\hline Technique & $60(11.5)$ & $52(13.6)$ \\
\hline Test/service/blank & $15(2.9)$ & $32(8.4)$ \\
\hline \multicolumn{3}{|l|}{ Chest } \\
\hline Artifact & $187(15.6)$ & $150(17.8)$ \\
\hline Clipped anatomy & $42(3.5)$ & $15(1.8)$ \\
\hline Duplicate & $165(13.7)$ & $48(5.7)$ \\
\hline Motion & $106(8.8)$ & $78(9.2)$ \\
\hline Positioning error & $465(38.7)$ & 351 (41.6) \\
\hline Technique & $201(16.7)$ & $149(17.7)$ \\
\hline Test/service/blank & $36(3.0)$ & $53(6.3)$ \\
\hline \multicolumn{3}{|l|}{ Knee } \\
\hline Artifact & $30(9.1)$ & $12(17.1)$ \\
\hline Clipped anatomy & $12(3.6)$ & $1(1.4)$ \\
\hline Duplicate & $27(8.2)$ & $5(7.1)$ \\
\hline Motion & $32(9.7)$ & $13(18.6)$ \\
\hline Positioning error & $110(33.2)$ & $15(21.4)$ \\
\hline Technique & $111(33.5)$ & $23(32.9)$ \\
\hline Test/service/blank & $9(2.7)$ & $1(1.4)$ \\
\hline \multicolumn{3}{|l|}{ Lumber spine } \\
\hline Artifact & $43(13.8)$ & $9(14.5)$ \\
\hline Clipped anatomy & $9(2.9)$ & $0(0.0)$ \\
\hline Duplicate & $28(9.0)$ & $1(1.6)$ \\
\hline Motion & $105(33.7)$ & $17(27.4)$ \\
\hline Positioning error & $73(23.4)$ & $20(32.3)$ \\
\hline Technique & $48(15.9)$ & $12(19.4)$ \\
\hline Test/service/blank & $9(1.9)$ & $3(4.8)$ \\
\hline \multicolumn{3}{|l|}{ Pelvic } \\
\hline Artifact & $8(11.3)$ & $3(7.0)$ \\
\hline Clipped anatomy & $1(1.4)$ & $0(0.0)$ \\
\hline Duplicate & $11(15.5)$ & $2(4.7)$ \\
\hline Motion & $13(18.3)$ & $9(20.9)$ \\
\hline Positioning error & $26(36.6)$ & $22(51.2)$ \\
\hline Technique & $10(14.1)$ & $6(14.0)$ \\
\hline Test/service/blank & $2(2.8)$ & $1(2.3)$ \\
\hline
\end{tabular}

pandemic, with rejection rates of $39.8 \%$ and $42.7 \%$, and $19.2 \%$ and $17.2 \%$, respectively. However, the significant difference has found in positioning errors, duplicate, clipped anatomy and test/service/blank with $\mathrm{p}$-value $<0.05$.
While Table 2 shows the various reasons for rejection involving body parts. Majority of rejection reasons were positioning errors for both in PC and DC periods for the abdomen, chest, pelvis, and so on. Technique errors were the most common reason for rejection of the knee. Lastly, motion errors were the most common reason for rejection of the lumbar spine.

Figure 1 presents rejection rates for body parts with rates higher than the mean calculated for the institute. All body organs have higher rejection rate during the PC period except for the pelvis, which could suggest that those body parts are considered difficult in positioning.

Discussion. During COVID-19 Saudi Arabia followed WHO guidelines in applying extra precautionary measures such as lockdown except for urgent matter such as close of schools and converting to online education, mask wearing, and social distancing. In medical settings all clinics were restricted, virtual clinics were implemented in governmental and private hospitals, and only emergency operation were performed. ${ }^{8,9}$

In radiology departments, a clear instruction was given to the staff aiming to limit patients risks and exposures to the virus like wearing a mask and being screened before entering the department. Patients who are known or suspected to have COVID their images would be cancelled, and they are directed to emergency department taking in consideration infection control precautions. ${ }^{11}$ COVID-19 pandemic has markedly impacted routine medical services, there was a significant reduction in endoscopy volume, and most procedures performed were emergency treatments. ${ }^{12}$ Cancellation of elective and non-urgent surgeries have enabled surgeons to become critical staff resources for health systems in the treatment of COVID-19 patients. This caused a reduction in the number of scans performed. ${ }^{13}$ Currently, there is no published studies focusing on digital radiography reject rates DC period in KSA.

Our study was conducted using 6 DR machines, the reduction in images obtained between the 2 periods were due to the safety measurements implemented at our institute following $\mathrm{MOH}$ guidelines. We found that there is a reduction in total number of images from 23,861 to 15,376 images DC. Also the rejection rate we calculated DC was $11.4 \%$ this was significantly lower compared to $14.1 \%$ in the PC period, our repeated ratio was significantly similar to the findings of Alahmadi et al. ${ }^{14}$ However, it was marked higher than the percentage reported by Yurt et $\mathrm{al}^{2}$ with a rejection rate of $1.2 \%$ and reported by Atkinson et $\mathrm{al}^{15}$ with a rejection rate of $9 \%$. 
The reduction in the rejection rate DC may be due to the reduced number of scans performed daily, which provided the staff more time to set up the patient correctly. In addition, both staff and patients attempted to reduce the time spent in close contact, which had an impact on getting an accurate image on the first shot, also the absence of trainees and undergraduate students in the hospital mandate the staff to operate machine themselves and not worry on the training and education.

There were several reasons for image rejection. The most frequent was positioning errors in both periods. This was similar to the findings of the studies by Yurt et $\mathrm{al}^{2}$ in 2018, Atkinson et $\mathrm{al}^{15}$ in 2019, and Alahmadi et $\mathrm{al}^{14}$ in 2019 . Another prominent reason in this study was technique errors.

Study limitations. As there was some data loss due to machine upgrades after September 2020. Second, identification of the technologist with the highest rejection rate is of great value; however, this was not performed in our study due to only one user account being logged into the machines to speed up the process of scanning.

In conclusion, this study shows that the rejection rate DC pandemic has significantly decreased in comparison to the PC period in our institute, but still higher than the recommended rate by WHO. It has also revealed that the radiographers cause more than $50 \%$ of the repeated scans. Which could explain this reduction as the radiographers being more cautious with the patient's position to decrease the time of contact. In addition, the reduced flow scans DC might have given the radiographers more time to position the patient and reduce rejected images.

We recommend the implementation of quality training for radiographers and investigating new protocols to optimize and decrease the rejection rate.

Acknowledgment. We would like to acknowledge Dr. Mohammed A. Safhi for his assistance with the statistical analysis. We would like to thank the Editage (www.editage.com)

Received 3rd March 2021. Accepted 7th July 2021.

From the Department of Radiology (Jastaniyyah, Khafaji, Albahiti), from the Faculty of Medicine (Jawhari, Mughrbel), King Abdulaziz University Hospital, Jeddah, Kingdom of Saudi Arabia.

Address correspondence and reprints request to: Dr. Abdulkarim A. Jawhari, Faculty of Medicine, King Abdulaziz University, Jeddah, Kingdom of Saudi Arabia. E-mail: Kremjohari@gmail.com ORCID ID: https://orcid.org/0000-0002-8470-7788

\section{References}

1. Gali RL, Roth CG, Smith E, Dave JK, editors. Does transitioning from computed radiography (CR) to direct radiography (DR) with portable imaging systems affect workflow efficiency? Proceedings Vol. 10579. Medical Imaging Informatics for Healthcare, Research, and Applications; 2018 March 6. SPIE, Houston (TX). p. 34.

2. Yurt A, Tintas M, Ramazan Y. Reject analysis in digital radiography: A prospective study. Int J Anat Radiol Surg 2018; 7: 31-34.

3. Hui SCN, Pialasse JP, Wong JYH, Lam T ping, Ng BKW, Cheng JCY, et al. Radiation dose of digital radiography (DR) versus micro-dose $\mathrm{x}$-ray (EOS) on patients with adolescent idiopathic scoliosis: 2016 SOSORT- IRSSD "John Sevastic Award" Winner in Imaging Research. Scoliosis Spinal Disord 2016; 11: 46.

4. Bernier MO, Baysson H, Pearce MS, Moissonnier M, Cardis E, Hauptmann M, et al. Cohort profile: The EPI-CT study: A European pooled epidemiological study to quantify the risk of radiation-induced cancer from paediatric CT. Int J Epidemiol 2019; 48: 379g-381g.

5. Hill KD, Einstein AJ. New approaches to reduce radiation exposure. Trends Cardiovasc Med 2016; 26: 55-65.

6. Alyousef K, Alkahtani S, Alessa R, Alruweili H. Radiographs reject analysis in a large tertiary care hospital in Riyadh. Glob J Qual Saf Healthc 2019; 2: 30.

7. World Health Organization. Radiation and Health. [Updated 2021. Cited 2021 Jun 12]. Available from: https://www.who. int/teams/environment-climate-change-and-health/radiationand-health

8. World Health Organization. Archived: WHO Timeline COVID-19. [Updated 2020 June 29. Cited 2021 Jan 27]. Available from: https://www.who.int/news/item/27-04-2020who-timeline---covid-19

9. World Health Organization. Impact of COVID-19 on people's livelihoods, their health and our food systems. [Updated 2020 Oct 13. Cited 2021 Jan 27]. Available from: https://www.who. $\mathrm{int} /$ news/item/13-10-2020-impact-of-covid-19-on-people'slivelihoods-their-health-and-our-food-systems

10. Centers for Disease Control and Prevention. Interim Infection Prevention and Control Recommendations for Healthcare Personnel During the Coronavirus Disease 2019 (COVID-19) Pandemic. [Updated 2019. Cited 2021 Jun 11]. Available from: https://www.cdc.gov/coronavirus/2019-ncov/hcp/infectioncontrol-recommendations.html

11. Ministry of Health. COVID 19 Guidelines. Radiology Departments Preparedness Plan for COVID-19. [Updated 2020. Cited 2021 Jun 11]. Available from: https://www. moh.gov.sa/Ministry/MediaCenter/Publications/Documents/ Radiology-Departments-Preparedness.pdf

12. Alboraie M, Piscoya A, Tran QT, Mendelsohn RB, Butt AS, Lenz $\mathrm{L}$, et al. The global impact of COVID-19 on gastrointestinal endoscopy units: An international survey of endoscopists. Arab J Gastroenterol 2020; 21: 156-161.

13. Al-Jabir A, Kerwan A, Nicola M, Alsafi Z, Khan M, Sohrabi C, et al. Impact of the Coronavirus (COVID-19) pandemic on surgical practice - Part 1. Int J Surg 2020; 79: 168-179.

14. Alahmadi O, Alrehaili A, Gameraddin M. Evaluation of reject analysis of chest radiographs in diagnostic radiology. Am J Diagnostic Imaging 2019; 5: 4.

15. Atkinson S, Neep M, Starkey D. Reject rate analysis in digital radiography: an Australian emergency imaging department case study. J Med Radiat Sci 2020; 67: 72-79. 